Document downloaded from:

http://hdl.handle.net/10251/99984

This paper must be cited as:

Guirao Sánchez, AJ.; Montesinos Santalucia, V. (2015). A note on the Mackey-star topology on a dual Banach space. Revista de la Real Academia de Ciencias Exactas Físicas y Naturales Serie A Matemáticas. 109(2):417-418. doi:10.1007/s13398-014-0192-4

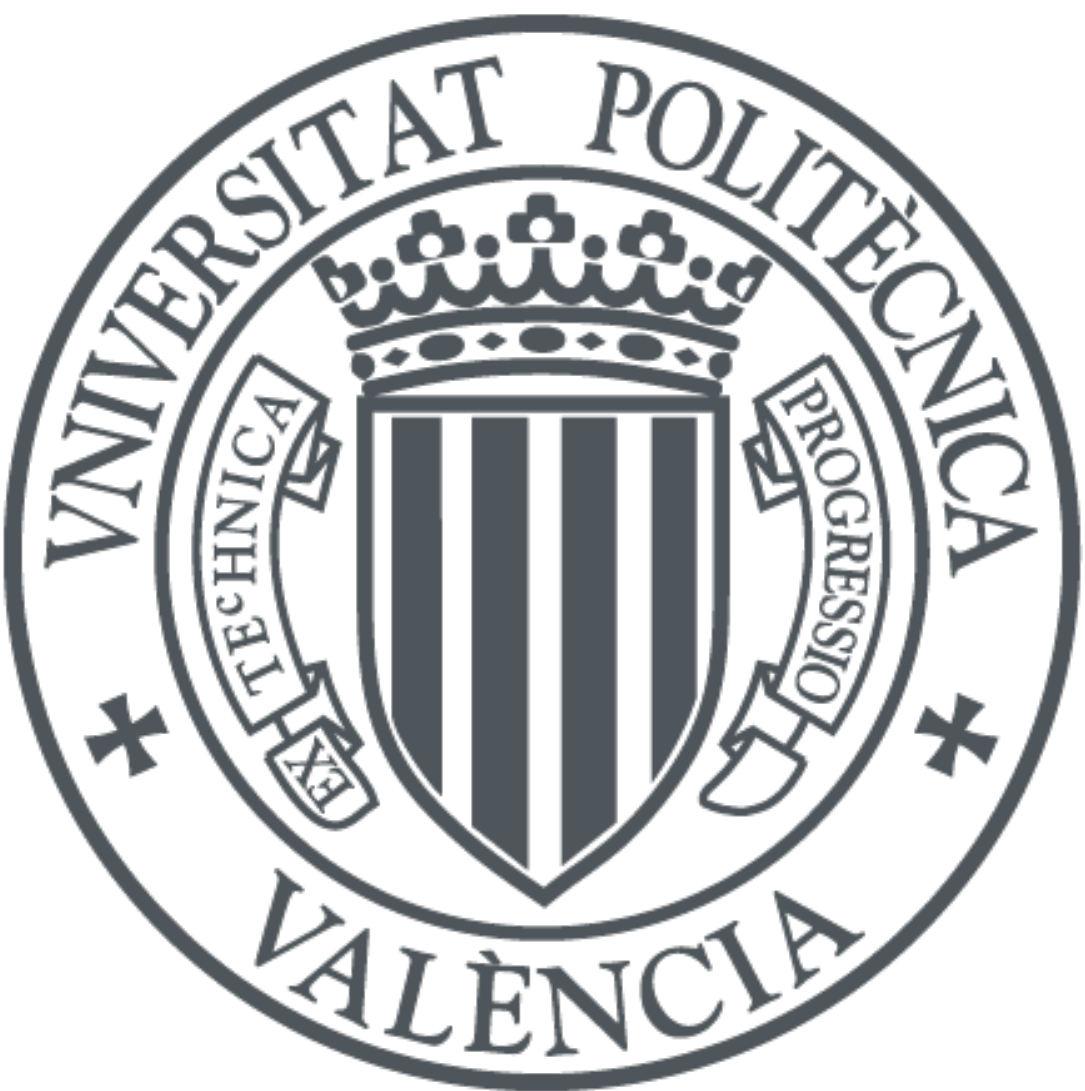

The final publication is available at

http://doi.org/10.1007/s13398-014-0192-4

Copyright Springer-Verlag

Additional Information 


\title{
A note on the Mackey-star topology on a dual Banach space
}

\author{
A. J. Guirao, V. Montesinos ${ }^{\dagger}$
}

March 24, 2018

\begin{abstract}
By using a result in R. B. Kirk, A note on the Mackey topology for $\left(C^{b}(X)^{*}, C^{b}(X)\right)$, Pacific Journal of Mathematics, 45, 2 (1973), 543-554, we show that there are separable Banach spaces such that their dual spaces, endowed with the Mackey-star topology, are not analytic. This solves a question raised in J. Kąkol, W. Kubiś, M. López-Pellicer, Descriptive Topology in Selected Topics of Functional Analysis, Springer Verlag, 2012, and in J. Kąkol, M. López-Pellicer, On realcompact topological vector spaces, RACSAM 105 (2011), 39-70.
\end{abstract}

Let $X$ be a Banach space. The topology Mackey-star (denoted $\mu^{*}$ ) on $X^{*}$ is the topology of the uniform convergence on the family of all (convex and balanced) weakly compact subsets of $X$ (see, e.g., [Ko, $\S 21.4]$, where this topology is denoted by $\boldsymbol{\mathfrak { d }}_{k}(X)$ ). It is a simple consequence of the Grothendieck completeness criterium (see, e.g., [Gr, $2 . \S 14])$ that $\left(X^{*}, \mu^{*}\right)$ is always complete. A Banach space $X$ is said to be strongly weakly compactly generated (SWCG, in short) (see $[\mathrm{SW}]$ ) whenever there exists a weakly compact subset $K_{0}$ of $X$ such that, for every weakly compact subset $K$ of $X$ and for every $\varepsilon>0$ there exists $n \in \mathbb{N}$ such that $K \subset n K_{0}+\varepsilon B_{X}$. Examples of SWCG Banach spaces include the reflexive ones, the separable Schur spaces, and $L^{1}(\mu)$, where $\mu$ is a $\sigma$-finite measure (see, e.g., [SW] and [FMZ]). It is simple to prove that a Banach space $X$ is SWCG if, and only if, $\left(B_{X^{*}}, \mu^{*}\right)$ is metrizable. This shows, in particular, that a separable Banach space $X$ is SWCG if, and only if, $\left(B_{X^{*}}, \mu^{*}\right)$ is a Polish space.

A topological space is said to be analytic if it is the continuous image of a Polish space (alternatively, if it is the continuous image of the space $\mathbb{N}^{\mathbb{N}}$ ). Analytic spaces are clearly separable. Even more, they are hereditarily separable.

\footnotetext{
* Universidad Politécnica de Valencia. Instituto de Matemática Pura y Aplicada. Camino de Vera, $\mathrm{s} / \mathrm{n}, 46022$ Valencia, Spain. Supported in part by MICINN and FEDER (project MTM2008-05396), by Fundación Séneca (project 08848/PI/08), by Generalitat Valenciana (GV/2010/036), and by Universidad Politécnica de Valencia (project PAID-06-09-2829). email: anguisa2@mat.upv.es

${ }^{\dagger}$ Universidad Politécnica de Valencia. Instituto de Matemática Pura y Aplicada. Camino de Vera, s/n, 46022 Valencia, Spain. Supported in part by Project MICINN MTM2011-22417, Generalitat Valenciana (GV/2010/036), and by Universidad Politécnica de Valencia (project PAID-06-09-2829). email: vmontesinos@mat.upv.es.

AMS classification: Primary: 46B10. Secondary: 54H05. Keyword and phrases: analytic space, Mackeystar topology, strongly weakly compactly generated space, Banach space.
} 
In [KLP, Prop. 18], and in [KKLP, Prop. 6.14], the following result is stated:

(*) Let $X$ be a SWCG Banach space. Then $\left(X^{*}, \mu^{*}\right)$ is analytic if, and only if, $X$ is separable.

In the notation above, the necessary condition follows from the fact that $K_{0}$, endowed with the restriction of the weak topology, is separable. That the condition is sufficient follows from the fact, that if $X$ is separable and SWCG, then $\left(B_{X^{*}}, \mu^{*}\right)$ is a Polish space.

In [KLP, p. 61], and in [KKLP, p. 170], the authors raise the following question:

(Q) Let $X$ be a separable Banach space. Is it true that $\left(X^{*}, \mu^{*}\right)$ is an analytic space?

We show here that the answer to this question is negative. For this purpose, it is enough to use the following result (note that a completely regular topological space $T$ is isomorphic to a subspace of $\left(X^{*}, w^{*}\right)$, where $(X,\|\cdot\|):=\left(C^{b}(T),\|\cdot\|_{\infty}\right)$, i.e., the space of all continuous and bounded real-valued functions on $T$, endowed with the supremum norm $\left.\|\cdot\|_{\infty}\right)$.

Theorem 1 (Kirk, [Kr]) Let $(T, \mathcal{T})$ be a completely regular topological space. Then (i) The topologies $\mathcal{T}$ and $\mu^{*}$ coincide on $T$ if, and only if, $\mathcal{T}$ is discrete.

(ii) The space $\left(T, \mu^{*}\right)$ is totally disconnected.

(iii) If $\mathcal{T}$ is first countable, then $\mu^{*}$ on $T$ is discrete.

Consider now the separable Banach space $(X,\|\cdot\|):=\left(C(K),\|\cdot\|_{\infty}\right)$, where $K$ is an uncountable compact metric space. According to Theorem 1 , the space $\left(K, \mu^{*}\right)$ is discrete (and so it cannot be separable). It follows from the remark above that the space $\left(X^{*}, \mu^{*}\right)$ is not analytic. This solves in the negative question (Q). In view of the result

(*) above, the space $X$ is not SWCG.

A Banach space $X$ has the property that $\left(X^{*}, \mu^{*}\right)$ is analytic if, and only if, $\left(B_{X^{*}}, \mu^{*}\right)$ is analytic. Indeed, every closed subspace of an analytic space is also analytic, so $\left(B_{X^{*}}, \mu^{*}\right)$ is analytic if $\left(X^{*}, \mu^{*}\right)$ is. In the other direction, it is enough to observe that a countable union of analytic subspaces of a topological space is analytic.

Note that $\left(B_{X^{*}}, \mu^{*}\right)$ is analytic if $X$ is a separable Asplund Banach space. Indeed, the identity mapping $I:\left(B_{X^{*}},\|\cdot\|\right) \rightarrow\left(B_{X^{*}}, \mu^{*}\right)$ is continuous, and $\left(B_{X^{*}},\|\cdot\|\right)$ is a Polish space, since $\left(X^{*},\|\cdot\|\right)$ is separable.

\section{References}

[FMZ] M. Fabian, V. Montesinos, and V. Zizler, On weak compactness in $L_{1}$ spaces. Rocky Mountains J. Math. 39 (2009), 1885-1893.

[Gr] A. Grothendieck, Topological Vector Spaces, Gordon and Breach, 1973.

[KLP] J. Kąkol, M. López-Pellicer, On realcompact topological vector spaces, RACSAM 105 (2011), 39-70. 
[KKLP] J. Kąkol, W. Kubiś, M. López-Pellicer, Descriptive Topology in Selected Topics of Functional Analysis. Springer Verlag, 2012.

[Kr] R. B. Kirk, A note on the Mackey topology for $\left(C^{b}(X)^{*}, C^{b}(X)\right)$, Pacific Journal of Mathematics, 45, 2 (1973), 543-554.

[Ko] G. Köthe, Topological Vector Spaces I, Springer Verlag, 1969.

[SW] G. Schlüchtermann and R. F. Wheeler, On strongly WCG Banach spaces, Math. Z. 199 (1988), 387-398. 